PROCEEDINGS OF THE

AMERICAN MATHEMATICAL SOCIETY

Volume 135, Number 7, July 2007, Pages 2089-2093

S 0002-9939(07)08858-2

Article electronically published on February 2, 2007

\title{
ON REGULARIZATION OF PLURISUBHARMONIC FUNCTIONS ON MANIFOLDS
}

\author{
ZBIGNIEW BŁOCKI AND SŁAWOMIR KOŁODZIEJ
}

(Communicated by Mei-Chi Shaw)

\begin{abstract}
We study the question of when a $\gamma$-plurisubharmonic function on a complex manifold, where $\gamma$ is a fixed $(1,1)$-form, can be approximated by a decreasing sequence of smooth $\gamma$-plurisubharmonic functions. We show in particular that it is always possible in the compact Kähler case.
\end{abstract}

\section{INTRODUCTION}

Let $M$ be a complex manifold. Recall that a function $\varphi: M \rightarrow \mathbb{R} \cup\{-\infty\}$ is called quasi-plurisubharmonic if it can be locally written as a sum of a plurisubharmonic and a smooth function. For a continuous $(1,1)$-form $\gamma$ on $M$ by $P S H(M, \gamma)$ we will denote the class of quasi-plurisubharmonic functions $\varphi$ on $M$ such that $d d^{c} \varphi+\gamma \geq 0$. Note that constants belong to $\operatorname{PSH}(M, \gamma)$ if $\gamma \geq 0$. On the other hand, the class $\operatorname{PSH}(M, \gamma)$ is always closed under the operations of maximum and regularized maximum (see e.g. Lemma I-5.18 in [3]).

Classes of $\gamma$-plurisubharmonic functions on manifolds appear in various geometric problems (see e.g. 12] or [10]). The case of a compact Kähler manifold $(M, \omega)$ is especially interesting. Guedj and Zeriahi [6] used complicated methods developed by Demailly (see [2], 4], [5]) to show that such an approximation is possible when $M$ admits a positive holomorphic line bundle.

We will show that in the arbitrary compact Kähler case such an approximation is possible without any loss of positivity. This implies in particular that the assumption in [7, that the form is Hodge, is superfluous. More generally, we will prove the following.

Theorem 1. Let $\omega$ be a positive continuous $(1,1)$-form on a compact manifold $M$. Then for every $\varphi \in \operatorname{PSH}(M, \omega)$ there exists a sequence $\varphi_{j} \in P S H(M, \omega) \cap C^{\infty}(M)$ decreasing to $\varphi$.

Theorem 1 will be an easy consequence of the following result which can in fact be deduced from Theorem 2.3 in [5] or Theorem 3.2 in [4] (and their proofs).

Theorem 2. Let $M$ be a complex manifold with a positive hermitian form $\omega$. Assume that $\gamma$ is a continuous $(1,1)$-form on $M$ and let $\varphi \in P S H(M, \gamma)$ be such

Received by the editors March 8, 2006

2000 Mathematics Subject Classification. Primary 32U05, 32Q15, 32U25.

Key words and phrases. Plurisubharmonic functions, Kähler manifolds, Lelong numbers.

Both authors were partially supported by KBN Grant \#2 P03A 03726. The second author was also supported by the Rector of the Jagiellonian University Fund. 
that the Lelong number $\nu_{\varphi}(z)=0$ for all $z \in M$ (this is, for example, the case when $\varphi$ is locally bounded). Then for every open $M^{\prime} \subset M$ we can find $\varepsilon_{j} \downarrow 0$ and $\varphi_{j} \in \operatorname{PSH}\left(M^{\prime}, \gamma+\varepsilon_{j} \omega\right) \cap C^{\infty}\left(M^{\prime}\right)$ decreasing to $\varphi$ in $M^{\prime}$.

Proof of Theorem 1. Without loss of generality we may assume that $\varphi \leq-1$. Since $\omega$ is positive, we have a sequence $\max \{\varphi,-j\} \in P S H(M, \omega) \cap L^{\infty}(M)$ decreasing to $\varphi$. We may thus also assume that $\varphi$ is bounded. By Theorem 2 we can then find $\lambda_{j} \downarrow 1$ and negative $\psi_{j} \in P S H\left(M, \lambda_{j} \omega\right) \cap C^{\infty}(M)$ decreasing to $\varphi$ (note that since $M$ is compact we may take $M^{\prime}=M$ in Theorem 2). Then one can easily check that $\varphi_{j}:=\psi_{j} / \lambda_{j} \in \operatorname{PSH}(M, \omega) \cap C^{\infty}(M)$ is a sequence decreasing to $\varphi$.

In fact, Theorem 2 is only a very special case of Theorem 3.2 in [4. The methods used to prove this result, developed originally in [2, are rather complicated. They involve local approximation of plurisubharmonic functions by holomorphic functions from weighted Bergman spaces and use in particular the Hörmander and Ohsawa-Takegoshi $L^{2}$-estimates for the $\bar{\partial}$-operator (in [6] this method is applied globally for sections of a positive holomorphic line bundle). On the other hand, if $\varphi$ is continuous, then approximation by smooth functions is easy (see [11] and Lemma 2.15 in [2]). Moreover, this method of gluing functions from different charts also works if the local approximating sequences are arbitrarily uniformly close to each other on intersection of charts. We show that this is the case for standard regularizations of plurisubharmonic functions provided that the Lelong numbers vanish (see Lemma 4 below). Thanks to this we obtain a simple, elementary proof of Theorem 2 (and thus of Theorem 1).

The assumption on the vanishing of the Lelong numbers in Theorem 2 is necessary. To show this we will more closely analyze an example from [6] let $\pi$ : $M \rightarrow \mathbb{C}^{2}$ be the blow-up of $\mathbb{C}^{2}$ at the origin. One can then show that there exists a smooth form $\gamma$ on $M$ such that $\pi_{*} \gamma=-d d^{c} \log |z|$ in $\mathbb{C}^{2} \backslash\{0\}$ (in fact $\gamma=[E]-\pi^{*} d d^{c} \log |z|$, where $[E]$ is the current of integration along the exceptional divisor $\left.E:=\pi^{-1}(0)\right)$. Moreover, there is uniquely defined Kähler form $\omega$ on $M$ such that $\pi_{*} \omega=d d^{c}\left(|z|^{2}+\log |z|\right)$ in $\mathbb{C}^{2} \backslash\{0\}$. Then, if $\varphi \in P S H(U, \gamma+\varepsilon \omega)$, where $U$ is a neighborhood of $E$, it follows that $\varphi \circ \pi^{-1}+\varepsilon|z|^{2}-(1-\varepsilon) \log |z|$ is plurisubharmonic in $\pi(U) \backslash\{0\}$, in particular it is bounded above near the origin. We conclude that $\varphi$ cannot be locally bounded if $\varepsilon<1$.

One of possible applications of Theorem 1 is a comparison principle for the complex Monge-Ampère operator. For continuous functions the proof of the following result is quite simple (see [9]) and in the general case, using Theorem 1, one can essentially repeat the original proof from [1] for domains in $\mathbb{C}^{n}$.

Theorem 3. For $\varphi, \psi \in P S H(M, \omega) \cap L^{\infty}(M)$, where $(M, \omega)$ is a compact Kähler manifold, one has

$$
\int_{\{\varphi<\psi\}}\left(d d^{c} \psi+\omega\right)^{n} \leq \int_{\{\varphi<\psi\}}\left(d d^{c} \varphi+\omega\right)^{n} .
$$

\section{Proof of Theorem 2}

We are now going to prove Theorem 2 using much simpler methods than in 2] (but of course we also get much less than in [2]). In the flat case we have the standard smooth regularization by convolution: if $\rho(z)=\widehat{\rho}(|z|) \in C_{0}^{\infty}\left(\mathbb{C}^{n}\right)$ is such 
that $\widehat{\rho} \geq 0, \widehat{\rho}(r)=0$ for $r \geq 1, \int_{\mathbb{C}^{n}} \rho d \lambda=1, \rho_{\delta}(z):=\delta^{-2 n} \rho(z / \delta)$ for $\delta>0$, we set

$$
u_{\delta}(z)=\left(u * \rho_{\delta}\right)(z)=\int u(z-\delta w) \rho(w) d \lambda(w) .
$$

Then, if $u$ plurisubharmonic, $u_{\delta}$ is decreasing to $u$ as $\delta \downarrow 0$. On the other hand, if $u$ is continuous, then $u_{\delta} \rightarrow u$ locally uniformly.

The following lemma will be crucial.

Lemma 4. Assume that $U, V \subset \mathbb{C}^{n}$ are open and let $F: U \rightarrow V$ be a biholomorphic mapping. Let $u \in P S H(U)$ be such that $\nu_{u}(z)=0$ for all $z \in U$. Define $u_{\delta}^{F}:=$ $\left(u \circ F^{-1}\right)_{\delta} \circ F$. Then $u_{\delta}-u_{\delta}^{F}$ tends locally uniformly to 0 as $\delta \rightarrow 0$.

Proof. We will first prove it for a different kind of approximation:

$$
\widehat{u}_{\delta}(z):=\max _{\bar{B}(z, \delta)} u \text {. }
$$

If $u$ is plurisubharmonic, then $\widehat{u}_{\delta}(z)$ is logarithmically convex in $\delta$, and from this we can easily deduce that $\widehat{u}_{\delta}$ is continuous, plurisubharmonic in $U_{\delta}:=\{z \in U$ : $\bar{B}(z, \delta) \subset U\}$ and decreases to $u$ as $\delta \downarrow 0$. If for example $a \geq 1$, then for a fixed $r>0$ and $\delta$ sufficiently small by the logarithmic convexity

$$
0 \leq \widehat{u}_{a \delta}-\widehat{u}_{\delta} \leq \frac{\log a}{\log (r / \delta)}\left(\widehat{u}_{r}-\widehat{u}_{\delta}\right) .
$$

This implies that if the Lelong numbers vanish, then for any positive constant $a$ we have locally uniform convergence

$$
\widehat{u}_{a \delta}-\widehat{u}_{\delta} \rightarrow 0
$$

as $\delta \rightarrow 0$.

We also set

that is,

$$
\widehat{u}_{\delta}^{F}:=\left(\widehat{u \circ F^{-1}}\right)_{\delta} \circ F
$$

$$
\widehat{u}_{\delta}^{F}(z)=\max _{F^{-1}(\bar{B}(F(z), \delta))} u .
$$

For a fixed $K \subset U$ we can find $A>1$ such that for $z \in K$ and $\delta$ sufficiently small

$$
\bar{B}(F(z), \delta) \subset F(\bar{B}(z, A \delta)), \quad F(\bar{B}(z, \delta)) \subset \bar{B}(F(z), A \delta) .
$$

Then on $K$ we have $\widehat{u}_{\delta}^{F} \leq \widehat{u}_{A \delta}, \widehat{u}_{\delta} \leq \widehat{u}_{A \delta}^{F}$, and combining this with (1) we get that $\widehat{u}_{\delta}^{F}-\widehat{u}_{\delta}$ tends locally uniformly to 0 . To complete the proof it is enough to use the following lemma.

Lemma 5. Assume that $u \in P S H(U)$ is such that $\nu_{u}(z)=0$ for every $z \in U$. Then $\widehat{u}_{\delta}-u_{\delta} \rightarrow 0$ locally uniformly in $U$ as $\delta \rightarrow 0$.

Proof. Consider another regularization in terms of spherical means:

$$
\widetilde{u}_{\delta}(z):=\frac{1}{\sigma(\partial B(z, \delta))} \int_{\partial B(z, \delta)} u d \sigma,
$$

where $d \sigma$ is the area measure on $\partial B(z, \delta)$. It is known that $\widetilde{u}_{\delta}(z)$ is increasing and logarithmically convex in $\delta$. We have $u_{\delta} \leq \widetilde{u}_{\delta} \leq \widehat{u}_{\delta}$ and

$$
u_{\delta}(z)=\int_{0}^{1} \widetilde{u}_{t \delta}(z) \widetilde{\rho}(t) d t,
$$


where

$$
\widetilde{\rho}(t)=\sigma(\partial B(0,1)) t^{2 n-1} \widehat{\rho}(t)
$$

(so that in particular $\int_{0}^{1} \widetilde{\rho}(t) d t=1$ ). Using the Poisson kernel for subharmonic functions one can show (see e.g. [8]) that

$$
0 \leq \widehat{u}_{\delta}-\widetilde{u}_{\delta} \leq \frac{3^{2 n-1}}{2^{2 n-2}}\left(\widehat{u}_{\delta}-\widehat{u}_{\delta / 2}\right)
$$

Since the Lelong numbers vanish, by (1) we get $\widehat{u}_{\delta}-\widetilde{u}_{\delta} \rightarrow 0$ locally uniformly. Finally, using logarithmic convexity, for some fixed $r>0$ and $\delta$ small enough we have

$$
0 \leq \widetilde{u}_{\delta}-u_{\delta}=\int_{0}^{1}\left(\widetilde{u}_{\delta}-\widetilde{u}_{t \delta}\right) \widetilde{\rho}(t) d t \leq \int_{0}^{1} \frac{\log (1 / t)}{\log (r / t \delta)}\left(\widetilde{u}_{r}-\widetilde{u}_{t \delta}\right) \widehat{\rho}(t) d t .
$$

Again, similarly as before, we conclude that $\widetilde{u}_{\delta}-u_{\delta} \rightarrow 0$ locally uniformly.

Remark. The assumption on the vanishing of the Lelong numbers in Lemmas 4 and 5 is necessary: if for example $u(z)=\log |z|$ and $F(z)=a z$, then $\widehat{u}_{\delta}(0)=\log \delta$, $\widehat{u}_{\delta}^{F}(0)=\log \delta-\log |a|, u_{\delta}(0)=\log \delta+\int_{0}^{1} \widehat{\rho}(t) \log t d t$, and $u_{\delta}^{F}(0)=u_{\delta}(0)-\log |a|$.

Proof of Theorem 2. Fix $\varepsilon>0$. We can then find a finite number of charts $V_{\alpha} \subset U_{\alpha}$ such that $\left\{V_{\alpha}\right\}$ is a covering of $M^{\prime}$ and

$$
0 \leq d d^{c} f_{\alpha}-\gamma \leq \varepsilon \omega \text { in } U_{\alpha}
$$

for some smooth functions $f_{\alpha}$ in $U_{\alpha}$. Then $u_{\alpha}:=\varphi+f_{\alpha}$ is plurisubharmonic in $U_{\alpha}$, and, if by $u_{\alpha, \delta}^{F}$ we denote the regularization of $u_{\alpha}$ in $U_{\beta}$, then by Lemma 4

$$
u_{\alpha, \delta}-u_{\beta, \delta}=u_{\alpha, \delta}-u_{\alpha, \delta}^{F}+\left(u_{\alpha}-u_{\beta}\right)_{\delta}^{F} \rightarrow f_{\alpha}-f_{\beta}
$$

locally uniformly in $U_{\alpha} \cap U_{\beta}$ as $\delta \rightarrow 0$. Let $\eta_{\alpha}$ be smooth in $U_{\alpha}$ and such that $\eta_{\alpha}=0$ in $V_{\alpha}$ and $\eta_{\alpha}=-1$ away from a compact subset of $U_{\alpha}$. We have $d d^{c} \eta_{\alpha} \geq-C \omega$ for some constant $C$ (depending on $\varepsilon$ ). Set

$$
\varphi_{\delta}=\max _{\alpha}\left(u_{\alpha, \delta}-f_{\alpha}+\varepsilon \eta_{\alpha} / C\right) .
$$

By (3), if $\delta$ is sufficiently small, the values on the sets $\left\{\eta_{\alpha}=-1\right\}$ do not contribute to the maximum, and thus $\varphi_{\delta}$ is continuous. If we consider regularized maximum instead of maximum in the above definition we can get smooth $\varphi_{\delta}$. Using (2) we obtain $\varphi_{\delta} \in P S H\left(M^{\prime}, \gamma+2 \varepsilon \omega\right)$. It also clear that $\varphi_{\delta}$ decreases to $\varphi$ as $\delta \downarrow 0$. From this we will easily find $\varepsilon_{j} \downarrow 0$ and $\varphi_{j} \in P S H\left(M^{\prime}, \gamma+\varepsilon_{j} \omega\right) \cap C^{\infty}\left(M^{\prime}\right)$ decreasing to $\varphi$.

\section{REFERENCES}

[1] E. Bedford, B.A.Taylor, A new capacity for plurisubharmonic functions, Acta Math. 149 (1982), 1-41. MR0674165 (84d:32024)

[2] J.-P. Demailly, Regularization of closed positive currents and intersection theory, J. Alg. Geom. 1 (1992), 361-409. MR1158622 (93e:32015)

[3] J.-P. Demailly, Complex Analytic and Differential Geometry, 1997, see http://www-fourier. ujf-grenoble.fr $/ \sim$ demailly/books.html.

[4] J.-P. Demailly, M. Paun, Numerical characterization of the Kähler cone of a compact Kähler manifold, Ann. of Math. 159 (2004), 1247-1274. MR.2113021 (2005i:32020)

[5] J.-P. Demailly, T, Peternell, M. Schneider, Pseudo-effective line bundles on compact Kähler manifolds, Internat. J. Math. 12 (2001), 689-741. MR1875649 (2003a:32032)

[6] V. Guedj, A.Zeriahi, Intrinsic capacities on compact Kähler manifolds, J. Geom. Anal. 15 (2005), 607-639. MR2203165 (2006j:32041) 
[7] V. Guedj, A.Zeriahi, Monge-Ampère operators on compact Kähler manifolds, see http://arxiv.org/PS_ cache/math/pdf/0504/0504234.pdf.

[8] C.O. Kiselman, Attenuating the singularities of plurisubharmonic functions, Ann. Pol. Math. 60 (1994), 173-197. MR1301603 (95i:32024)

[9] S. Kołodziej, Stability of solutions to the complex Monge-Ampère equation on compact Kähler manifolds, Indiana Univ. Math. J 52 (2003), 667-686. MR1986892 (2004i:32062)

[10] D.H.Phong, J.Sturm, The Monge-Ampère operator and geodesics in the space of Kähler potentials, Invent. Math. 166 (2006), 125-149. MR.2242635

[11] R. Richberg, Stetige streng pseudokonvexe Funktionen, Math. Ann. 175 (1968), 257-286. MR0222334 (36:5386)

[12] S.-T. Yau, On the Ricci curvature of a compact Kähler manifold and the complex MongeAmpère equation, I, Comm. Pure Appl. Math. 31 (1978), 339-411. MR0480350(81d:53045)

Institute of Mathematics, Jagiellonian University, Reymonta 4, 30-059 Kraków, POLAND

E-mail address: Zbigniew.Blocki@im.uj.edu.pl

Institute of Mathematics, Jagiellonian University, Reymonta 4, 30-059 Kraków, Poland

E-mail address: Slawomir.Kolodziej@im.uj.edu.pl 